\title{
Multifrequency variability of the blazar AO $0235+164^{\star}$ The WEBT campaign in 2004-2005 and long-term SED analysis
}

\author{
C. M. Raiteri ${ }^{1}$, M. Villata $^{1}$, M. Kadler ${ }^{2,3}$, M. A. Ibrahimov ${ }^{4}$, O. M. Kurtanidze ${ }^{5}$, V. M. Larionov ${ }^{6}$, M. Tornikoski ${ }^{7}$,
} P. Boltwood ${ }^{8}$, C.-U. Lee ${ }^{9}$, M. F. Aller ${ }^{10}$, G. E. Romero ${ }^{11,12}$, H .D. Aller ${ }^{10}$, A. T. Araudo ${ }^{11,12}$, A. A. Arkharov ${ }^{13}$, U. Bach ${ }^{1}$, D. Barnaby ${ }^{14}$, A. Berdyugin ${ }^{15}$, C. S. Buemi ${ }^{16}$, M. T. Carini ${ }^{14}$, D. Carosati ${ }^{17}$, S. A. Cellone ${ }^{12}$, R. Cool ${ }^{18}$, M. Dolci ${ }^{19}$, N. V. Efimova ${ }^{13}$, L. Fuhrmann ${ }^{1,20}$, V. A. Hagen-Thorn ${ }^{6}$, M. Holcomb ${ }^{14}$, I. Ilyin ${ }^{21}$, V. Impellizzeri ${ }^{2}$, R. Z. Ivanidze ${ }^{5}$, B. Z. Kapanadze ${ }^{5}$, J. Kerp ${ }^{22}$, T. S. Konstantinova ${ }^{6}$, Y. Y. Kovalev ${ }^{23,24,2}$, Yu. A. Kovalev ${ }^{24}$, A. Kraus ${ }^{2}$, T. P. Krichbaum ${ }^{2}$, A. Lähteenmäki ${ }^{7}$, L. Lanteri ${ }^{1}$, P. Leto $^{25}$, E. Lindfors ${ }^{15}$, J. R. Mattox ${ }^{26}$, N. Napoleone ${ }^{27}$, M. G. Nikolashvili ${ }^{5}$, K. Nilsson ${ }^{15}$, J. Ohlert ${ }^{28}$, I. E. Papadakis ${ }^{29,30}$, M. Pasanen ${ }^{15}$, C. Poteet ${ }^{14}$, T. Pursimo ${ }^{31}$, E. Ros ${ }^{2}$, L. A. Sigua ${ }^{32}$, S. Smith ${ }^{14}$, L. O. Takalo ${ }^{15}$, C. Trigilio ${ }^{16}$, M. Tröller ${ }^{7}$, G. Umana ${ }^{16}$, H. Ungerechts ${ }^{33}$, R. Walters ${ }^{14}$, A. Witzel ${ }^{2}$, and E. Xilouris ${ }^{34}$

(Affiliations can be found after the references)

Received 1 June 2006 / Accepted 16 August 2006

\section{ABSTRACT}

Aims. A huge multiwavelength campaign targeting the blazar AO 0235+164 was organized by the Whole Earth Blazar Telescope (WEBT) in 2003-2005 to study the variability properties of the source.

Methods. Monitoring observations were carried out at $\mathrm{cm}$ and $\mathrm{mm}$ wavelengths, and in the near-IR and optical bands, while three pointings by the XMM-Newton satellite provided information on the X-ray and UV emission.

Results. We present the data acquired during the second observing season, 2004-2005, by 27 radio-to-optical telescopes. The 2600 data points collected allow us to trace the low-energy behaviour of the source in detail, revealing an increased near-IR and optical activity with respect to the previous season. Increased variability is also found at the higher radio frequencies, down to $\sim 15 \mathrm{GHz}$, but not at the lower ones. While the X-ray (and optical) light curves obtained during the XMM-Newton pointings reveal no significant short-term variability, the simultaneous intraday radio observations with the $100 \mathrm{~m}$ telescope at Effelsberg show flux-density changes at $10.5 \mathrm{GHz}$, which are more likely due to a combination of intrinsic and extrinsic processes.

Conclusions. The radio (and optical) outburst predicted to peak around February-March 2004 on the basis of the previously observed 5-6 yr quasi-periodicity did not occur. The analysis of the optical light curves reveals now a longer characteristic time scale of variability of $\sim 8$ yr, which is also present in the radio data. The spectral energy distributions corresponding to the XMM-Newton observations performed during the WEBT campaign are compared with those pertaining to previous pointings of X-ray satellites. Bright, soft X-ray spectra can be described in terms of an extra component, which appears also when the source is faint through a hard UV spectrum and a curvature of the X-ray spectrum. Finally, there might be a correlation between the X-ray and optical bright states with a long time delay of about $5 \mathrm{yr}$, which would require a geometrical interpretation.

Key words. galaxies: active - galaxies: BL Lacertae objects: general - galaxies: BL Lacertae objects: individual: AO $0235+164$ - galaxies: jets galaxies: quasars: general

\section{Introduction}

Among the active galactic nuclei, blazars (i.e. flat-spectrum radio quasars and BL Lac objects) exhibit the most extreme properties: violent variability at all wavelengths, high optical (and radio) polarization, superluminal motion of radio components, and brightness temperatures often exceeding the inverse Compton limit. These features are explained by assuming that the emission comes from a relativistic plasma jet closely aligned with the line of sight (see e.g. Blandford \& Rees 1978; Blandford \& Königl 1979).

One of the most intriguing blazars is AO $0235+164$ $(\mathrm{J} 0238+1636)$ at $z=0.94$ which, in addition to the above

* For questions regarding the availability of the data presented in this paper, please contact the WEBT President Massimo Villata (villata@to.astro.it) characteristics, also shows a complex environment, where a couple of foreground galaxies at $z=0.524$, possibly interacting, can both significantly contaminate its emission in the optical-UV band and absorb its radiation from the near-IR to the soft X-rays. Moreover, the stars of these objects might act as microlenses, producing at least part of the observed variability (e.g. Ostriker \& Vietri 1985; Stickel et al. 1988).

The analysis of the radio and optical light curves of AO $0235+164$ from 1975 to 2000 revealed correlated variability and a quasi-periodicity of the main radio (and optical) outbursts on a $\sim 5.7$ yr time scale (Raiteri et al. 2001). The latter feature made this object a good candidate for the binary black hole scenario (Romero et al. 2003; Ostorero et al. 2004), as in the case of OJ 287, another famous blazar (e.g. Sillanpää et al. 1988; Villata et al. 1998). 
In 2003-2005 AO 0235+164 has been the target of a huge multiwavelength observing effort chiefly aimed at verifying its possible quasi-periodicity, since a radio outburst had been predicted to peak around February-March 2004 (with an uncertainty of 6 months). The Whole Earth Blazar Telescope ${ }^{1}$ (WEBT) consortium organized a radio, near-IR, and optical long-term monitoring campaign, during which three observations by the XMM-Newton satellite were performed, with simultaneous dense radio monitoring at the $100 \mathrm{~m}$ Effelsberg telescope. A spectroscopic monitoring was also carried out at the VLT, TNG, and NTT telescopes to study the relationship between the Mg II line flux and the continuum flux density, and 15 VLBA epochs were granted to study the source structure variability. The results of these observations will be presented elsewhere.

The results of the first observing season 2003-2004 were reported by Raiteri et al. (2005), while a detailed analysis of the X-ray observations was performed by Raiteri et al. (2006). Here we present the radio-to-optical data acquired by 27 telescopes during the second observing season, 2004-2005. The data of this extensive WEBT campaign 2003-2005, added to the historical light curves, allow one to study the behaviour of this source over the last $30 \mathrm{yr}$.

This paper is organized as follows: the optical, near-IR, and radio light curves obtained during the 2004-2005 observing season are presented in Sect. 2. In Sect. 3 we derive optical colour indices over a longer time interval, including the 1989-1991 and 1997-1998 outbursts, to study the spectral variability of the source. The intraday observations performed with the Effelsberg radio telescope during the August 2004 and January 2005 XMM-Newton pointings are presented and discussed in Sect. 4. The long-term optical and radio behaviour of AO $0235+164$ is presented in Sect. 5. In Sect. 6 we analyse 11 SEDs of the source corresponding to different epochs of X-ray observations, trying to understand how many emission components are involved. Finally, the conclusions are drawn in Sect. 7.

\section{Observations during the 2004-2005 season}

The second part of the WEBT campaign took place from May 1, 2004 (JD = 2453127 ) to May 1, 2005 (JD = 2453492 ). Table 1 shows the list of the participating observatories, with the size of the telescope, the frequency/wavelength/band, and the number of observations performed. As one can see, 2593 data points were acquired from 27 telescopes: 6 of them working at $\mathrm{cm}$ wavelengths, one at $\mathrm{mm}$ wavelengths, one in the near-IR bands, and 19 in the optical bands.

Since the source was often very faint, a number of data were affected by large errors. Hence, when constructing the light curves some data from the same telescope were binned ${ }^{2}$ to increase the signal to noise ratio, while some other data, in disagreement with the trend of the light curves, were discarded. This cleaning process was mainly performed on the optical light curves, where the comparison among the source behaviour in different bands and the better sampling can help to recognize unreliable points.

Moreover, since the presence of an AGN only 2 arcsec south of the source (named ELISA by Raiteri et al. 2005) affects the optical photometry of AO $0235+164$ especially in the blue part

\footnotetext{
${ }^{1}$ http://www.to.astro.it/blazars/webt/; see e.g. Villata et al. (2004a); Böttcher et al. (2005); Raiteri et al. (2005).

2 With binning interval not exceeding half an hour.
}

Table 1. Ground-based observatories participating in the 2004-2005 observing season of the WEBT campaign.

\begin{tabular}{lrcr}
\hline \hline Observatory & Tel. size & Bands & $N_{\text {obs }}$ \\
\hline SAO RAS (RATAN) & $600 \mathrm{~m}^{a}$ & $2.3,4.8,7.7$, & 33 \\
& & $11.1,21.7 \mathrm{GHz}$ & \\
Metsähovi & $14 \mathrm{~m}$ & $37 \mathrm{GHz}$ & 142 \\
Noto & $32 \mathrm{~m}$ & $5,8,22 \mathrm{GHz}$ & 56 \\
Medicina & $32 \mathrm{~m}$ & $5,8 \mathrm{GHz}$ & 23 \\
Effelsberg & $100 \mathrm{~m}$ & $4.9,10.5 \mathrm{GHz}$ & 68 \\
UMRAO & $26 \mathrm{~m}$ & $4.8,8.0,14.5 \mathrm{GHz}$ & 112 \\
Pico Veleta & $30 \mathrm{~m}$ & $1,2,3 \mathrm{~mm}$ & 18 \\
Campo Imperatore & $110 \mathrm{~cm}$ & $J, H, K$ & 154 \\
Bohyunsan & $180 \mathrm{~cm}$ & $V, R, I$ & 126 \\
Mt. Maidanak & $150 \mathrm{~cm}$ & $U, B, V, R, I$ & 733 \\
Abastumani & $70 \mathrm{~cm}$ & $R$ & 328 \\
Crimean & $70 \mathrm{~cm}$ & $B, V, R, I$ & 100 \\
Skinakas & $130 \mathrm{~cm}$ & $B, V, R, I$ & 83 \\
Tuorla & $103 \mathrm{~cm}$ & $R$ & 20 \\
Armenzano & $40 \mathrm{~cm}$ & $B, V, R, I$ & 87 \\
Michael Adrian & $120 \mathrm{~cm}$ & $R$ & 48 \\
Asiago & $182 \mathrm{~cm}$ & $B, V, R, I$ & 38 \\
Torino & $105 \mathrm{~cm}$ & $R, I$ & 20 \\
Roque (KVA) & $35 \mathrm{~cm}$ & $R$ & 69 \\
Roque (NOT) & $256 \mathrm{~cm}$ & $U, B, V, R, I$ & 24 \\
Roque (TNG) & $358 \mathrm{~cm}$ & $U, B, V, R, I$ & 10 \\
CASLEO & $215 \mathrm{~cm}$ & $B, V, R, I$ & 108 \\
La Silla (NTT) & $358 \mathrm{~cm}$ & $R$ & 5 \\
Boltwood & $40 \mathrm{~cm}$ & $R$ & 129 \\
Bell & $60 \mathrm{~cm}$ & $R$ & 23 \\
Kitt Peak (RCT) & $130 \mathrm{~cm}$ & $R$ & 24 \\
Kitt Peak (WIYN) & $90 \mathrm{~cm}$ & $B, V, R, I$ & 12 \\
\hline Total & & & 2593 \\
\hline
\end{tabular}

${ }^{a}$ Ring telescope.

of the spectrum, we subtracted its contribution as discussed in Raiteri et al. (2005).

\subsection{Optical and near-IR light curves}

Optical and near-IR data were collected as instrumental magnitudes of the source and of the reference stars in its field (Smith et al. 1985; Fiorucci et al. 1998) in order to apply the same magnitude calibration to all datasets, when possible.

The UBVRI light curves of the 2004-2005 observing season are shown in Fig. 1. Different symbols and colours are used to distinguish the provenance of the data, as described in the legend. Vertical lines are drawn through the dates of the XMM-Newton pointings of August 2, 2004 and January 28, 2005.

With respect to the previous observing season (see Raiteri et al. 2005 and the top panel of Fig. 3), in 2004-2005 the source showed increased variability, spanning more than $2.5 \mathrm{mag}$, although the average level has remained rather faint. In the $R$-band light curve, the best sampled one, four major flares can be recognized (around JD $=2453210,2453250,2453310$, and 2453390), which brought the source to be brighter than $R=17$. The most dramatic variations were observed during the rising and dimming phases of the second flare, when the $R$-band brightness first increased by $\sim 1.0 \mathrm{mag}$ in 2 days and then decreased by $\sim 2.2$ mag in 12 days.

Near-IR monitoring was performed at the Campo Imperatore Observatory in the $J H K$ bands. The resulting light curves are plotted in Fig. 2, compared to the $R$-band one. Although the near-IR light curves are less sampled than the $R$-band one, 


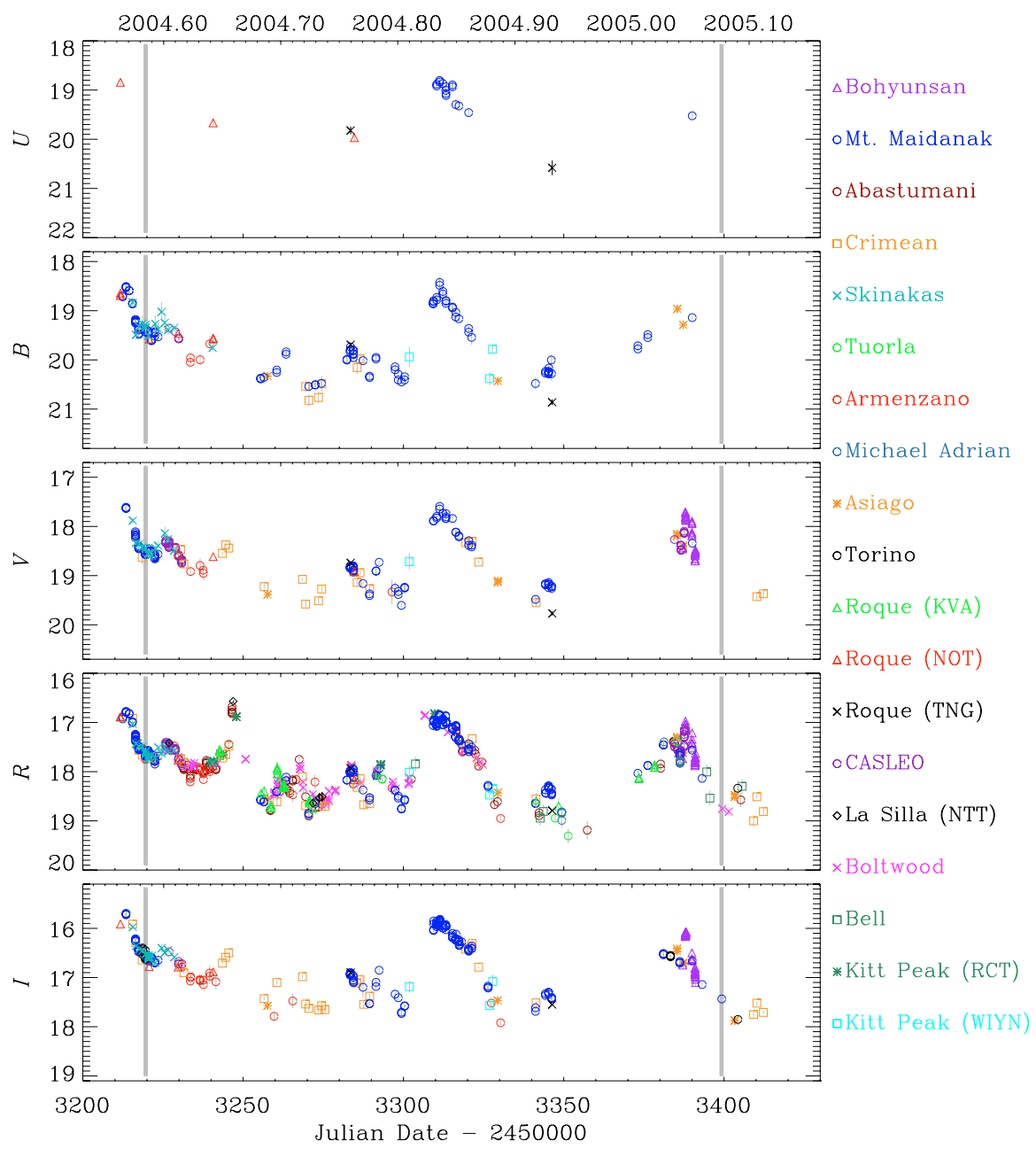

Fig. 1. UBVRI light curves of $\mathrm{AO} 0235+164$ during the observing season 2004-2005; vertical lines indicate the XMM-Newton pointings of August 2, 2004 and January 28, 2005. The ELISA contribution has been subtracted as explained in Raiteri et al. (2005). a general agreement is visible. In particular, it is possible to recognize the first flare around $\mathrm{JD}=2453210$ and the dip at $\mathrm{JD}=2453350$.

\subsection{Radio light curves}

A comparison between the $R$-band light curve and the radio behaviour at different wavelengths is displayed in Fig. 3 for both the observing seasons 2003-2004 and 2004-2005 of the WEBT campaign. Data from the VLA/VLBA Polarization Calibration Database are also shown ${ }^{3}$. The vertical lines mark the XMM-Newton pointings. Cubic splines through the 30-day binned light curve at $37 \mathrm{GHz}$ and 50-day binned light curves at $22,14.5,8$, and $5 \mathrm{GHz}$ are drawn to highlight the longterm variations ${ }^{4}$. Horizontal lines show the magnitude and fluxdensity average values over the considered period: $\langle R\rangle=17.84$, $\left\langle F_{37}\right\rangle=1.66 \mathrm{Jy},\left\langle F_{22}\right\rangle=1.32 \mathrm{Jy},\left\langle F_{14.5}\right\rangle=1.44 \mathrm{Jy},\left\langle F_{8}\right\rangle=$ $1.42 \mathrm{Jy},\left\langle F_{5}\right\rangle=1.49 \mathrm{Jy}$. These 2003-2005 optical and radio levels are relatively low if compared with those reached during the major historical outbursts; indeed, when looking at the historical light curves (Fig. 7) one can see that $R_{\min }=14.03$, $F_{37, \text { max }}=6.88 \mathrm{Jy}, F_{22, \text { max }}=6.10 \mathrm{Jy}, F_{14.5, \text { max }}=8.09 \mathrm{Jy}$, $F_{8, \text { max }}=7.03 \mathrm{Jy}$, and $F_{5, \text { max }}=4.28 \mathrm{Jy}$.

\footnotetext{
${ }^{3}$ http://www.vla.nrao.edu/astro/calib/polar/

${ }^{4}$ We chose a shorter time bin for the $37 \mathrm{GHz}$ data because the light curve at $37 \mathrm{GHz}$ is better sampled than those at the lower frequencies and flux density variations are faster.
}

As already found for AO $0235+164$ and for other blazars, variations have larger flux amplitudes at the higher frequencies. It is interesting to notice that a higher flux density is visible at $37 \mathrm{GHz}$ at the beginning of the 2004-2005 observing season, when the source was experiencing an optical flare. The radio flux density from 37 to $14.5 \mathrm{GHz}$ was also higher at the time of the optical flare which occurred around JD = 2453 300-2453 330, while at the start of the 2003-2004 season some radio activity at all frequencies seems to follow the bright optical state. However, it is hard to say whether these higher radio states have some correlation with their contemporaneous optical events, or if they are delayed counterparts of previous optical flares or, finally, if there is no correlation at all. Actually, each of these possibilities has already been singled out in the historical behaviour of the source (e.g. Raiteri et al. 2005, and references therein).

\section{Optical colour indices}

The colour behaviour of blazars can be an important tool to disentangle different components contributing to the optical emission variability. In the case of BL Lacertae, Villata et al. (2002, 2004b) showed that the short-term variations are strongly chromatic, with a bluer-when-brighter behaviour, likely due to intrinsic energetic processes. On the contrary, the longer-term flux changes do not imply important spectral variations, and are possibly of geometric origin. A recent study of the quasar-type blazar 3C 454.3 (Villata et al. 2006) shows that the optical spectrum steepens when the source is in outburst and the jet 

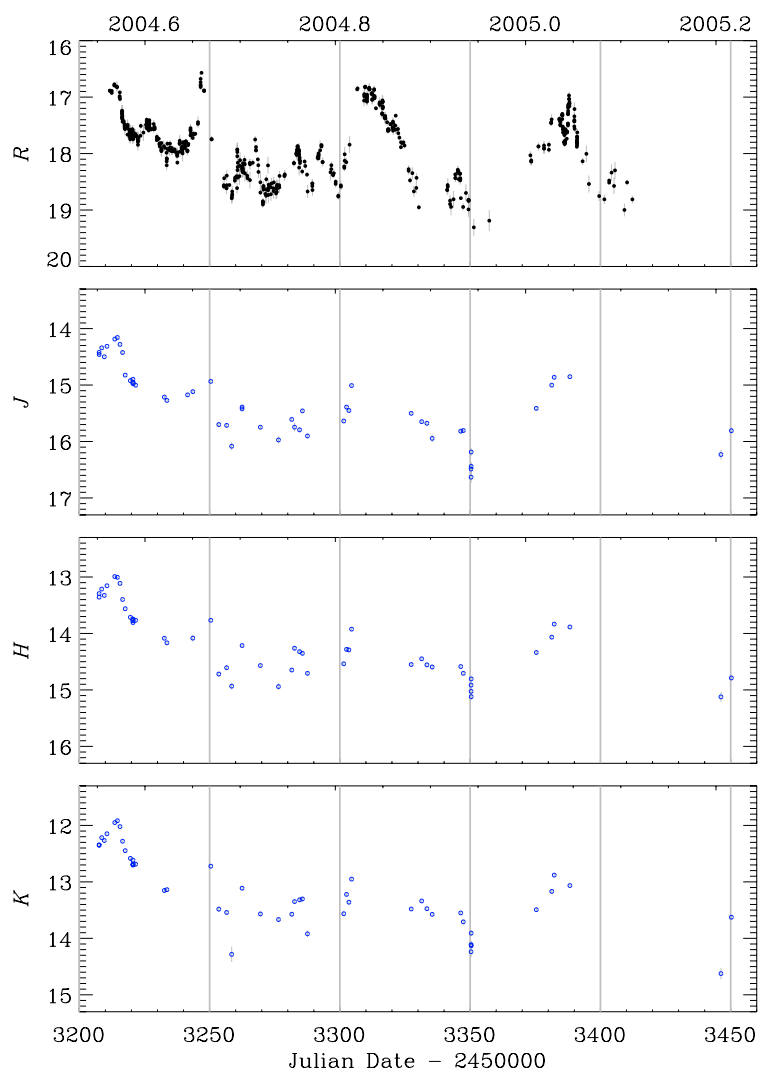

Fig. 2. JHK light curves of AO 0235+164 during the observing season 2004-2005 compared to the $R$-band one (top). Vertical lines are drawn to guide the eye through variations in different bands.

contribution to the optical flux is dominant. During fainter optical states instead, the emission from a blue thermal disc may be responsible for the observed spectral flattening (bluerwhen-fainter behaviour). The multi-colour optical observations of 8 blazars (3 quasars and 5 BL Lacs) by Gu et al. (2006) support this result, showing that quasars tend to be redder when they are brighter, while BL Lacs tend to be bluer. An analysis of the spectral behaviour of a sample of blazars was performed by Fiorucci et al. (2004): in particular, they found that the mean spectral slope is flatter during the rising phases of flares, while it is steeper during the declines.

We show the $B-R$ colour index of AO $0235+164$ as a function of time in Fig. 4 (bottom), compared to the $R$-band light curve (top). The plot covers the period 1989-2005 to include the last major optical outbursts. The $B-R$ points have been obtained by coupling each $B$ magnitude from a given telescope with the average of the $R$ data from the same telescope acquired next the $B$ datum (i.e. within 10-20 min). Only measurements with errors not greater than 0.08 mag were considered. In this way, we coupled $401 \mathrm{~B}$ data with $670 \mathrm{R}$ ones to obtain indices with a maximum error of $0.10 \mathrm{mag}$.

The colour index shows a noticeable scatter, from 1.27 to 2.07 , which implies important spectral variations. The average value is 1.73 , with a standard deviation of 0.11 . However, it is not possible to recognize any simple correlation with brightness. In particular, when considering the indices corresponding to the outburst phases of 1989-1991 and 1997-1998, they do not show peculiar values, since similar $B-R$ values are found also in much fainter states. Thus, it seems that the long-term behaviour of the source is essentially achromatic. The $V-I$ colour indices confirm this finding.
However, one can notice a progressive spectral reddening in the last years: although the average $R$ magnitude ${ }^{5}$ in the 2002-2003, 2003-2004, and 2004-2005 observing seasons is similar $(\sim 17.8)$, the average $B-R$ values are $1.66,1.72$, and 1.82 , respectively.

Finally, one can derive the optical spectral index $\alpha_{\text {opt }}\left(F_{v} \propto\right.$ $\left.v^{-\alpha}\right)$ from the colour index: $\alpha_{\text {opt }}=\left[(B-R)-\left(A_{B}-A_{R}\right)-\right.$ $0.3521] / 0.4125$. By setting the extinction values $A_{B}$ and $A_{R}$ equal to 1.904 and 1.260 , respectively (see Table 5 in Raiteri et al. 2005), we obtain an average value $\alpha_{\text {opt }}=1.76$, with standard deviation 0.26 . Although this value has to be taken with some caution because it is calculated from two bands only, it nonetheless gives an indication of the optical spectral behaviour of the source. The spectrum appears rather steep, but still within the range of spectral indices of low-energy peaked BL Lacs derived by Fiorucci et al. (2004). The very high values of $\alpha_{\mathrm{opt}}$ $(>2)$ estimated for AO $0235+164$ by various authors in previous works were obtained by neglecting the absorption due to the $z=0.524$ intervening system.

\section{Intraday radio observations at Effelsberg}

As in the case of the XMM-Newton pointing of January 18-19, 2004 discussed in Raiteri et al. (2005), also during the other two pointings of August 2, 2004 and January 28, 2005, densely sampled radio observations were performed at 4.9 and $10.5 \mathrm{GHz}$ with the $100 \mathrm{~m}$ radio telescope at Effelsberg. The observing strategy and data reduction procedures are based on Kraus et al. (2003) and have been outlined in Raiteri et al. (2005). The resulting light curves are shown in Fig. 5 for the August 2004 pointing and in Fig. 6 for the January 2005 one. The yellow areas highlight the period of the X-ray observations ${ }^{6}$. Normalized flux densities of the two calibrators 3C 067 (J0224+2750) (triangles) and $4 \mathrm{C}+09.11(\mathrm{~J} 0238+1010)$ (crosses) are shown in the bottom panels. Horizontal lines in both the source and the calibrators panels indicate $1 \%$ deviations from the mean flux densities. They allow to grab the amount of variability and to compare the source one with that of the calibrators.

In both epochs the $4.9 \mathrm{GHz}$ light curve does not show significant variability, while the flux density changes at $10.5 \mathrm{GHz}$ are more interesting. Indeed, on August 2, 2004 one can see first a sharp flux decrease of $3 \%$ in about $2 \mathrm{~h}^{7}$, and then a slower flux increase. On January 28, 2005 the percent variation of the source flux is not greater than the calibrators one, but the fact that we see a quasi-linear trend suggests that this can be a piece of a larger variation, and that the probability that it is noise is very low.

Table 2 reports some statistics: in both epochs the standard deviation $\sigma$ is greater than the root mean square uncertainty $\epsilon=\sqrt{\sum_{i=1}^{N} \epsilon_{i}^{2} / N}$ (where $\epsilon_{i}$ are the individual errors) at $10.5 \mathrm{GHz}$, while it is smaller at $4.9 \mathrm{GHz}$. The same conclusion is reached by considering the reduced chi-square $\chi_{\mathrm{r}}^{2}$, which tests the hypothesis that the light curve can be modelled by a constant

\footnotetext{
${ }^{5}$ Obtained by considering only the points which are used to calculate the colour indices.

6 The X-ray light curves corresponding to the three XMM-Newton pointings have been analysed in Raiteri et al. (2006); they show no significant variability. Further analysis of the radio and X-ray light curves can be found in Kadler (2005).

${ }^{7}$ Calculated as the variation between the first (the highest) and fifth (the lowest) point, which has also a slope equal to that determined by the second-fourth points. A similar, but more uncertain, slope is also simultaneously present in the $4.9 \mathrm{GHz}$ data.
} 

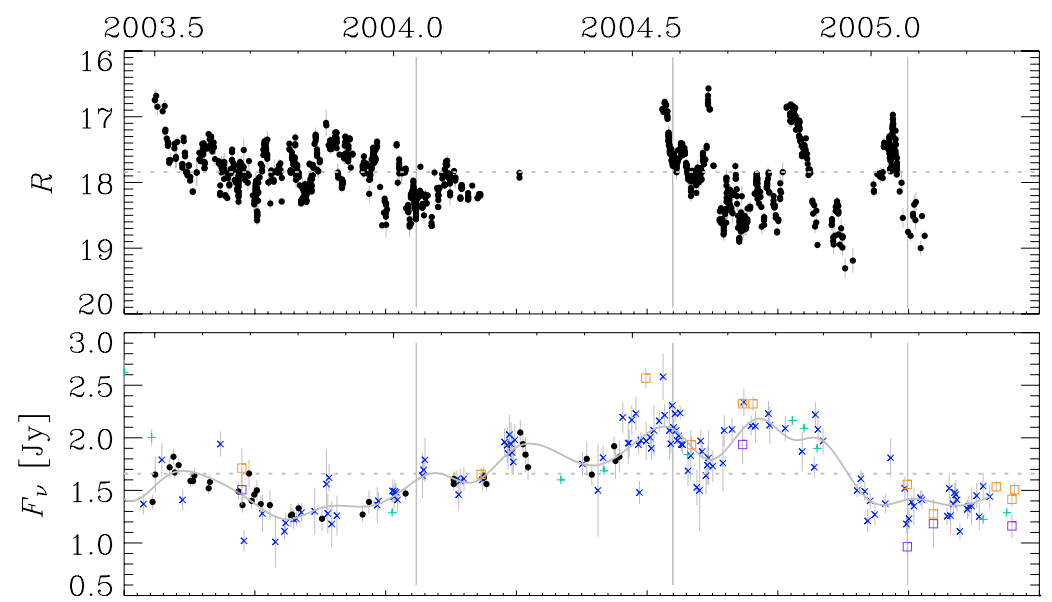

\section{$2 \mathrm{~mm}$, Pico Veleta $\square 3 \mathrm{~mm}$, Pico Veleta $+43 \mathrm{GHz}, \mathrm{VLA} /$ VLBA PCD \\ - $37 \mathrm{GHz}$, Metsahovi}

$\times 37 \mathrm{GHz}$, Metsahovi

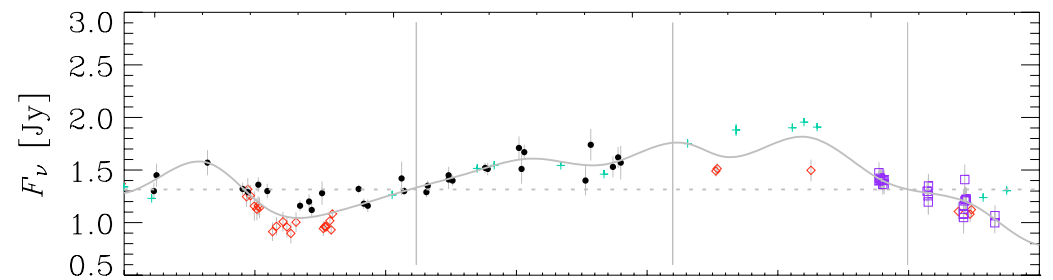

-22 GHz, Metsahovi

$\square 22 \mathrm{GHz}$, Noto

$+22 \mathrm{GHz}, \mathrm{VLA} / \mathrm{VLBA}$ PCD

21.7 GHz, RATAN

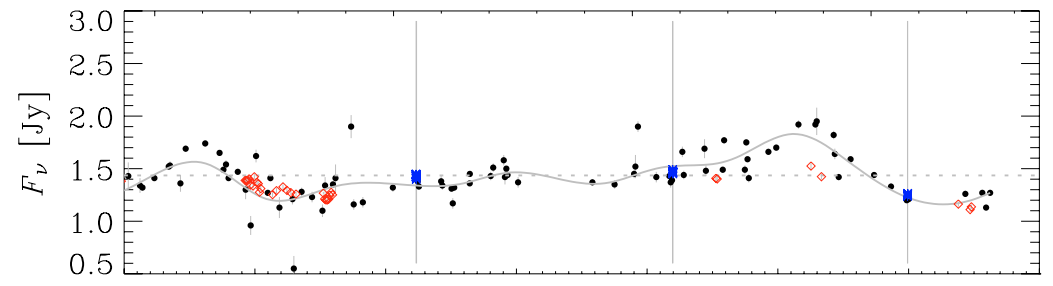

- $14.5 \mathrm{GHz}$, UMRAO

-11.1 GHz, RATAN

* $10.5 \mathrm{GHz}$, Effelsberg

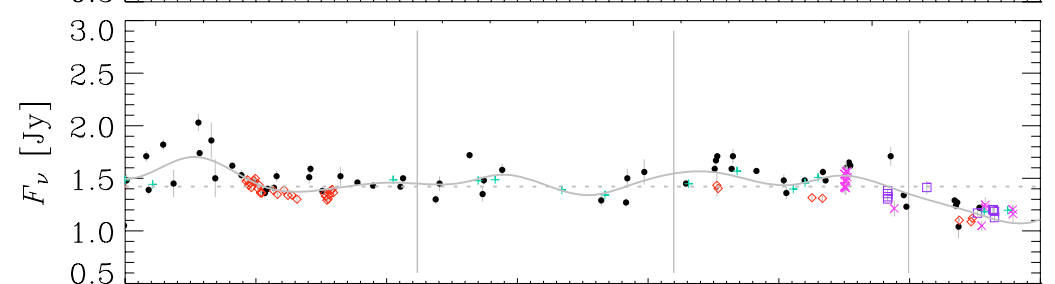

+ $8.5 \mathrm{GHz}, \mathrm{VLA} / \mathrm{VLBA}$ PCD

$\square 8 \mathrm{GHz}$, Noto

$\times 8 \mathrm{GHz}$, Medicina

- 8.0 GHz, UMRAO

$\bullet 7.7 \mathrm{GHz}, \mathrm{RATAN}$

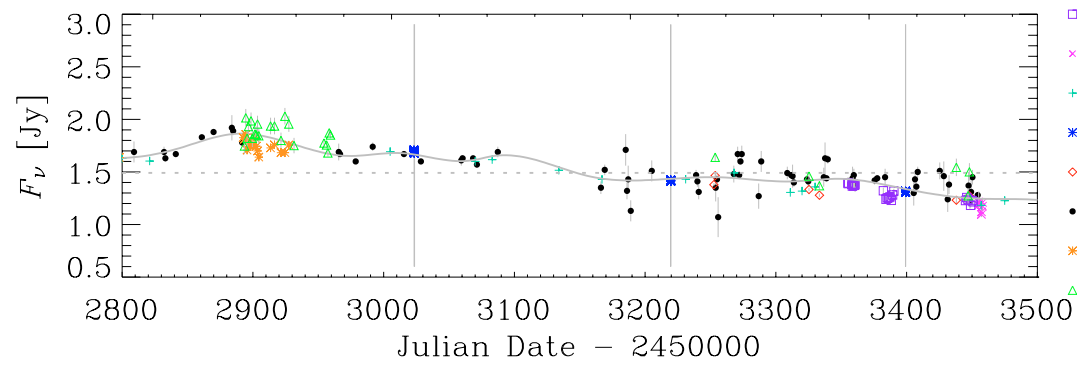

$\square 5 \mathrm{GHz}$, Noto

$\times 5 \mathrm{GHz}$, Medicina

$+5 \mathrm{GHz}, \mathrm{VLA} / \mathrm{VLBA}$ PCD

* $4.9 \mathrm{GHz}$, Effelsberg

$\diamond 4.8 \mathrm{GHz}, \mathrm{RATAN}$

- 4.8 GHz, UMRAO

*3.9 GHz, RATAN

$2.3 \mathrm{GHz}$, RATAN

Fig. 3. $R$-band magnitudes (top) and radio fluxes ( $\mathrm{Jy}$ ) at different frequencies during the 2003-2005 WEBT campaign. Vertical lines mark the times of the three XMM-Newton pointings. Cubic splines on the radio data at 37,22, 14.5, 8, and $5 \mathrm{GHz}$ are plotted to better see the long-term trend. Horizontal lines indicate the mean fluxes at these frequencies. Two different symbols are used for the $37 \mathrm{GHz}$ data from Metsähovi since they come from different datasets.

function: the values in Table 2 tell that this hypothesis is fair for the $4.9 \mathrm{GHz}$ data, but not for the $10.5 \mathrm{GHz}$ ones. Finally, Table 2 also shows the modulation index $m[\%]=100 \sigma /\left\langle F_{v}\right\rangle$, which provides a measure of the strength of the observed variation amplitudes without taking into account the errors of the individual measurements. This is lower at $4.9 \mathrm{GHz}$ than at $10.5 \mathrm{GHz}$ and inferior to what was found for the January 18-19, 2004 light curves, when the modulation index was $1.2 \%$ at $10.5 \mathrm{GHz}$ and $0.5 \%$ at $4.9 \mathrm{GHz}$.
The limited duration of the radio monitoring allows neither to fully constrain the true time scale of the $10.5 \mathrm{GHz}$ variations nor to check whether flux changes at $4.9 \mathrm{GHz}$ led or lagged those detected at the higher frequency. Hence, we are not able to say whether these flux changes are of intrinsic nature or if interstellar scattering is responsible for at least part of the variations. Also a higher modulation index at higher frequency may be reconciled with interstellar scintillation by invoking a strong scintillation regime (see e.g. Rickett 1990). 

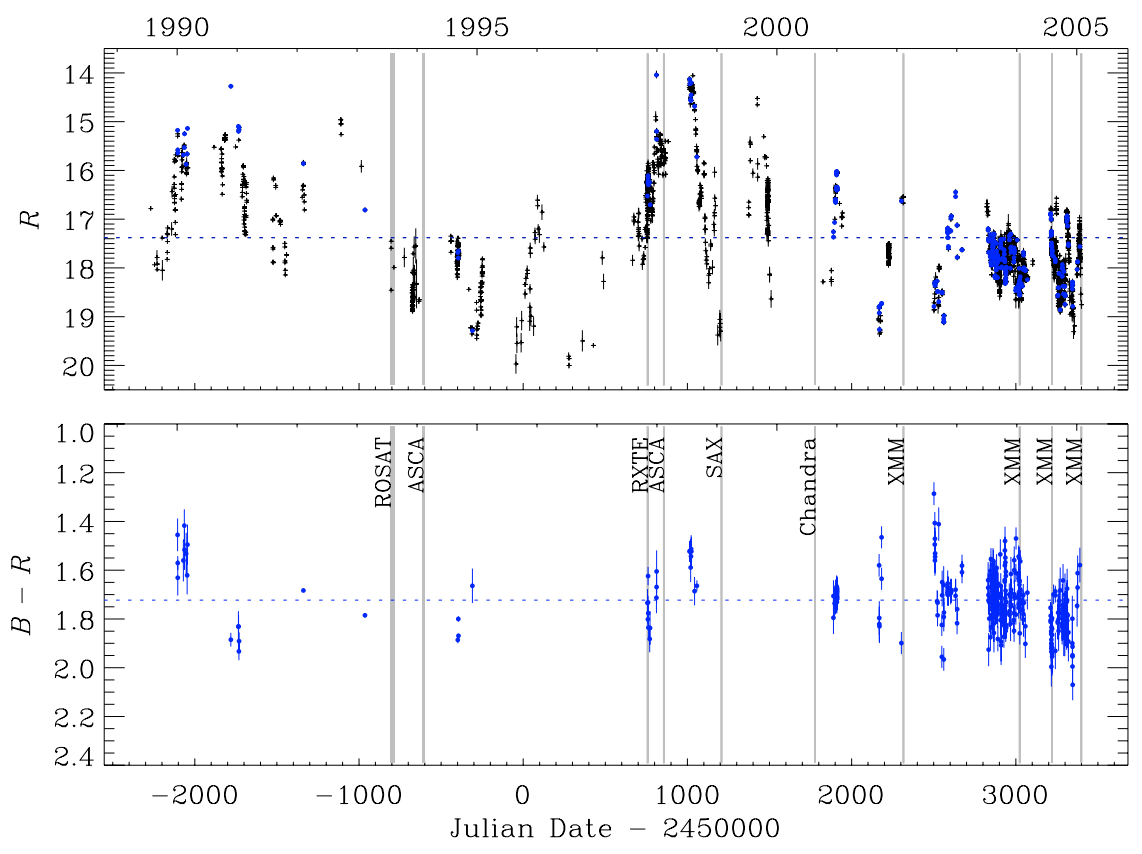

Fig. 4. $B-R$ colour indices (bottom) compared to the $R$-band light curve (top); in the top panel blue (larger) dots indicate the $R$ data used to derive the colour indices. Horizontal lines are drawn through average values; vertical lines indicate the times of the X-ray satellite pointings, discussed in Sect. 5.

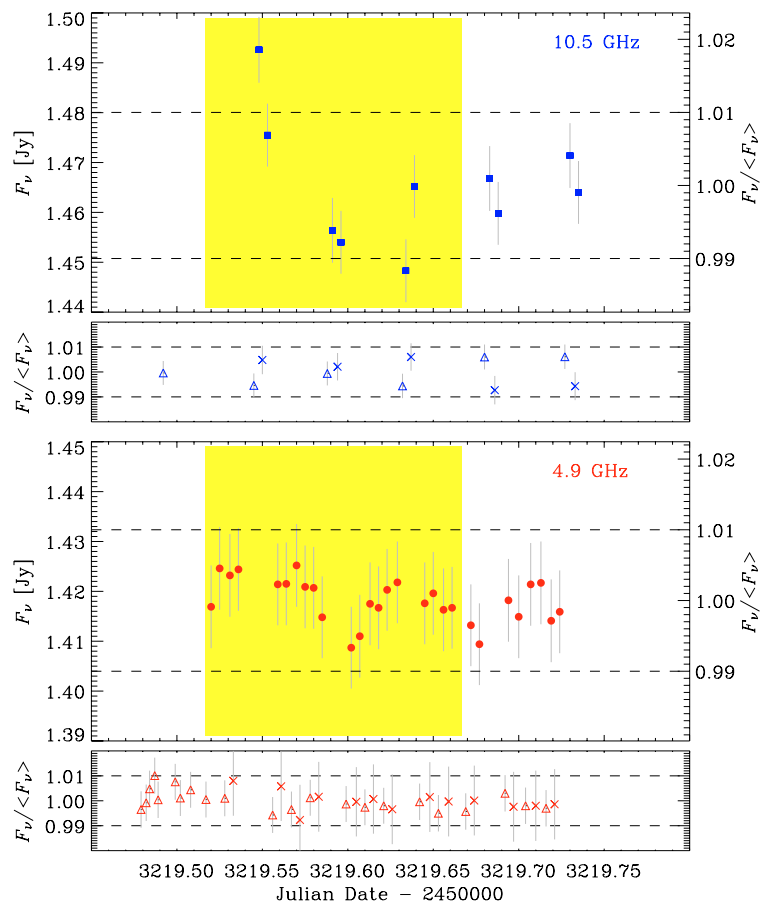

Fig. 5. Radio light curves of the source and calibrators at $10.5 \mathrm{GHz}$ (top, blue symbols) and $4.9 \mathrm{GHz}$ (bottom, red symbols) obtained with the $100 \mathrm{~m}$ radio telescope at Effelsberg on August 2, 2004. The two calibrators are $3 \mathrm{C} 067$ (triangles) and $4 \mathrm{C}+09.11$ (crosses). The horizontal lines indicate deviations of $1 \%$ from the mean flux densities, while the yellow areas highlight the period of the X-ray observations.

However, in the following we speculate about the physical implications of these variations in case we ascribed them to an intrinsic process.

Under the assumption of an intrinsic nature of the 10.5 $\mathrm{GHz}$ flux density variations detected in August 2, 2004, the brightness temperature corresponding to the initial fast decrease,

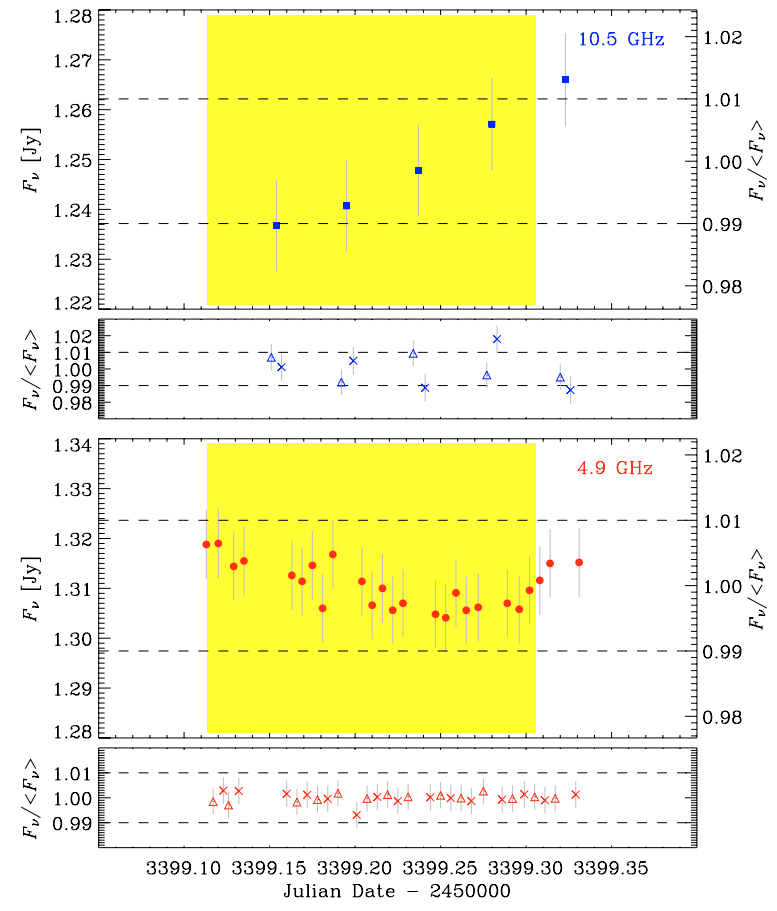

Fig. 6. Radio light curves of the source and calibrators at $10.5 \mathrm{GHz}$ (top, blue symbols) and $4.9 \mathrm{GHz}$ (bottom, red symbols) obtained with the $100 \mathrm{~m}$ radio telescope at Effelsberg on January 28, 2005. The two calibrators are 3C 067 (triangles) and 4C +09.11 (crosses). The horizontal lines indicate deviations of $1 \%$ from the mean flux densities, while the yellow areas highlight the period of the X-ray observations.

calculated following Wagner et al. $(1996)^{8}$, is $T_{\mathrm{b}} \sim 7 \times 10^{17} \mathrm{~K}$, and a Doppler factor $\delta>87$ would be required to bring the rest frame value below the inverse Compton limit of $10^{12} \mathrm{~K}$ $\left(T_{\mathrm{b}, \text { observed }}=\delta^{3} T_{\mathrm{b}, \text { intrinsic }}\right.$ ), confirming previous findings of a very high Doppler factor to explain some observing features of this source (Kraus et al. 1999; Fujisawa et al. 1999; Frey et al. 2000;

\footnotetext{
${ }^{8}$ We adopt a flat cosmology with $H_{0}=71 \mathrm{~km} \mathrm{~s}^{-1} \mathrm{Mpc}^{-1}$ and $\Omega=$ 0.27 , which yields a source luminosity distance of $\sim 6140 \mathrm{Mpc}$.
} 


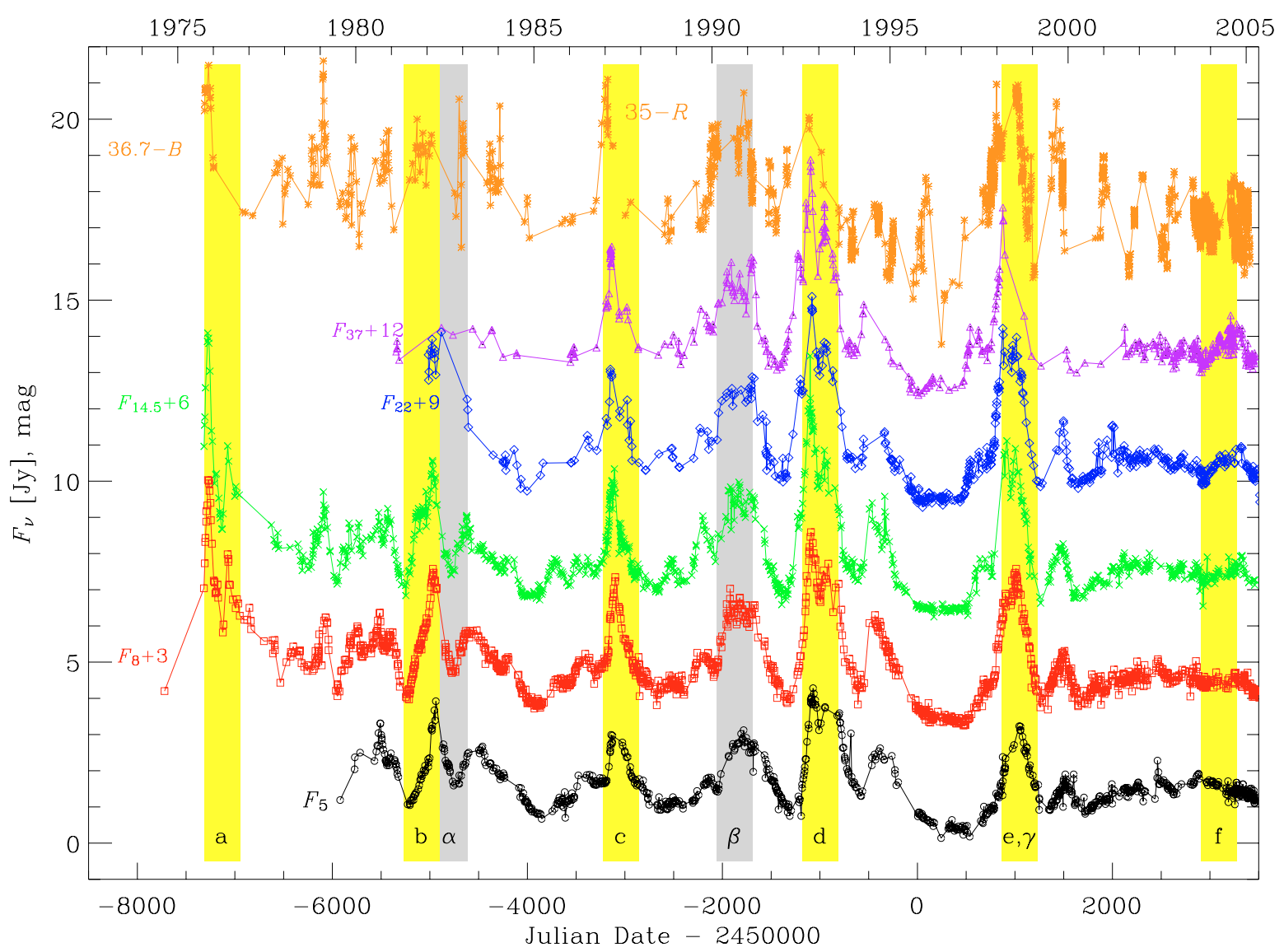

Fig. 7. Historical optical (mag) and radio $(37,22,14.5,8$, and $5 \mathrm{GHz}$, flux density in Jy) light curves of AO $0235+164$ shifted by the indicated amount; to increase the sampling in the optical, we show $R$-band data after JD $=2447000$ and $R$-converted $B$-band data before. Yellow strips highlight the periods (a, b, c, d, e, f) where a major radio (and optical) outburst is expected on the basis of a $5.6 \pm 0.5$ yr quasi-periodicity; periods $\alpha, \beta$, and $\gamma$ belong to an $\sim 8$ yr characteristic time scale of variability.

Table 2. Statistics on the Effelsberg data.

\begin{tabular}{lccccc}
\hline \hline $\begin{array}{l}\text { Freq. } \\
(\mathrm{GHz})\end{array}$ & $\begin{array}{c}\left\langle F_{v}\right\rangle \\
(\mathrm{Jy})\end{array}$ & $\begin{array}{c}\sigma \\
(\mathrm{Jy})\end{array}$ & $\begin{array}{c}\epsilon \\
(\mathrm{Jy})\end{array}$ & $\chi_{\mathrm{r}}^{2}$ & $\begin{array}{c}m \\
(\%)\end{array}$ \\
\hline \multicolumn{6}{c}{ August 2, 2004 } \\
10.5 & 1.465 & 0.013 & 0.006 & 3.76 & 0.89 \\
4.9 & 1.418 & 0.004 & 0.008 & 0.29 & 0.28 \\
\hline \multicolumn{5}{c}{ January 28, 2005 } \\
10.5 & 1.250 & 0.012 & 0.009 & 1.66 & 0.96 \\
4.9 & 1.311 & 0.005 & 0.007 & 0.45 & 0.38 \\
\hline
\end{tabular}

Jorstad et al. 2001). If we take the data errors into account, the inferred brightness temperature can actually vary from $\sim 3$ to $\sim 11 \times 10^{17} \mathrm{~K}$, corresponding to lower limits to $\delta$ of 70 and 104 , respectively.

On January 28 the $10.5 \mathrm{GHz}$ light curve shows a quasi-linear increasing trend, characterized by a $\sim 2 \%$ flux variation in about $4 \mathrm{~h}$. Under the assumption of intrinsic variability, the associated brightness temperature would be $T_{\mathrm{b}} \sim 9 \times 10^{16} \mathrm{~K}$, implying $\delta>45$. This value of the Doppler factor is similar to that estimated by Raiteri et al. (2005) from the Effelsberg observations of January $2004(\delta>46)$. Although it is much smaller than that derived from the August 2004 observations, nonetheless it would imply strong relativistic beaming of the emitted radiation in the intrinsic variability scenario. When considering the data errors, the lower limit to $\delta$ can actually range between 23 and 62 for this variation.
We have to mention that recent results, obtained during the current relatively quiescent state of the source, point to $\delta$ and $T_{\mathrm{b}}$ values lower than the extreme values estimated in the above mentioned earlier papers. Frey et al. (2006) analysed VSOP data acquired in 2001-2002. They found a decrease of the Doppler boosting by a factor of $\sim 20$ with respect to the value $\delta \sim 100$ inferred from data taken in 1999 just after a major outburst (Frey et al. 2000). Their results are consistent with those derived by Kovalev et al. (2005) from VLBA 2-cm Survey data of March 2001. Apparent speeds up to $(25.6 \pm 7) c$ were measured by Piner et al. (2006) in the jet of AO $0235+164$ based on VLBA observations during 2002-2003. These values are quite smaller than those previously reported by Jorstad et al. (2001) and suggest Doppler factors not exceeding $\sim 50$.

This boosting decrease can be explained, for instance, by an increased viewing angle of the radio emitting region in the helical jet scenario proposed by Villata \& Raiteri (1999) and applied to the case of AO $0235+164$ by Ostorero et al. (2004). In this case, the high brightness temperature value we inferred from the August 2 data would be overestimated and the observed variation would be due, at least in part, to interstellar scattering.

It is interesting to notice that the brightness temperature derived from VLBI observations performed on September 11, 2004 at $15 \mathrm{GHz}$ is only $2.6 \times 10^{11} \mathrm{~K}$ (MOJAVE collaboration, priv. comm.). Although both the VLBI and IDV brightness temperatures are lower limits, they are much more different than it is usually found for these compact objects (see e.g. Zensus et al. 2002), 


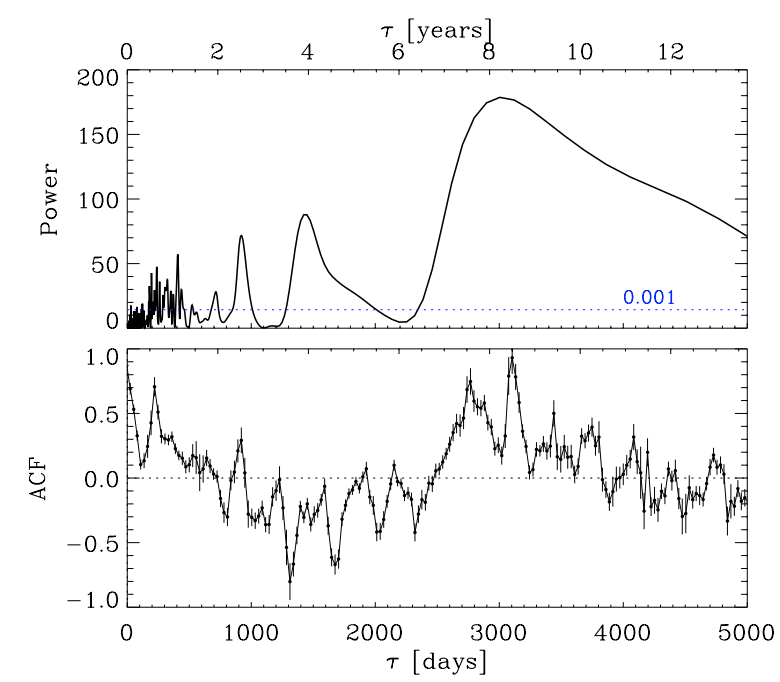

Fig. 8. Power spectrum of the discrete Fourier transform (top) and autocorrelation function (bottom) obtained from the historical $R$-band light curve. In the top panel the blue dotted line indicates the level above which the significance is better than 0.001 .

favouring an extrinsic explanation for the flux density variation observed on August 2, 2004.

\section{Long-term behaviour}

The historical behaviour of the optical magnitudes and radio fluxes is plotted in Fig. 7. These light curves have been shifted by a certain amount (indicated in the figure) to better distinguish the trend at different wavelengths. To increase the sampling in the optical, we plotted $R$-band data after JD $=2447000$ and $B$-band data converted into $R$ ones before, according to $R=B-\langle B-R\rangle$, with $\langle B-R\rangle=1.7$.

The five yellow strips named a, b, c, d, and e highlight the radio outbursts (with optical counterpart) which were found to repeat quasi-periodically by Raiteri et al. (2001). Indeed, the $1 \mathrm{yr}$ thick strips, equally spaced by $5.6 \mathrm{yr}$, contain the peak of the most prominent radio (and optical) outbursts. The last strip (f) indicates the period when the next outburst should have occurred according to the Raiteri et al. (2001) prediction, which motivated the WEBT campaign.

It is evident that the last $\sim 5 \mathrm{yr}$ of suppressed activity in the radio bands are very atypical for this source.

If we run a periodicity analysis on the historical optical light curves applying the discrete Fourier transform (DFT; Press et al. 1992), the most significant signal occurs at $\sim 3000$ days $(8.2 \mathrm{yr}$ ). A similar time scale can be found now also in the radio light curves, together with the 5-6 yr period found by Raiteri et al. $(2001)^{9}$. We show these results in Figs. 8 and 9.

Figure 8 (top panel) displays the results of the DFT applied to the historical $R$-band light curve. Before running the DFT algorithm, data have been binned over 1 day intervals to homogenize the sampling. The significance of peaks in the DFT, which correspond to sinusoidal components of period $\tau$ in the light

\footnotetext{
9 We point out that the present analysis is performed on different light curves with respect to Raiteri et al. (2001): not only they are more extended in time, but also more datasets have been added to both the optical and radio light curves to improve the sampling of the pre-2000 period, as discussed in Raiteri et al. (2005). In particular, besides the radio data from Metsähovi and UMRAO, we included data (both recent and past ones) from RATAN, Noto, Medicina, Effelsberg, and NRAO.
}

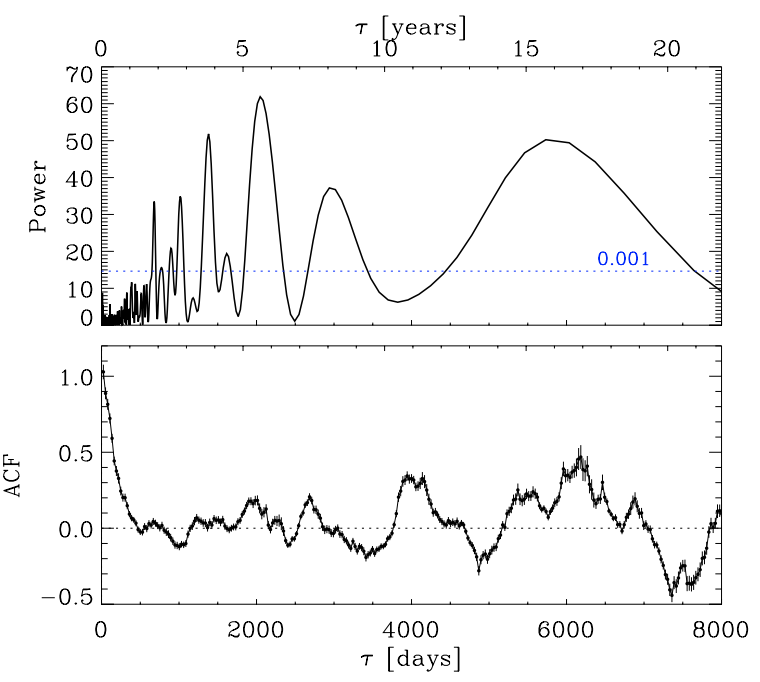

Fig. 9. Power spectrum of the discrete Fourier transform (top) and autocorrelation function (bottom) obtained from the historical $8 \mathrm{GHz}$ light curve. In the top panel the blue dotted line indicates the level above which the significance is better than 0.001 .

curve, is estimated by the false alarm probability, i.e. the probability that a peak is higher than a certain level if the data are pure noise. The horizontal (dotted blue) line in the figure marks the value of power corresponding to a significance level better than 0.001 . The DFT indicates that the most likely period is $8.2 \mathrm{yr}$, with other possible periods at 3.9 and $2.5 \mathrm{yr}$. The numerous signals corresponding to time scales around (or less than) $1 \mathrm{yr}$ may be spurious, since they may reflect gaps in the data train. The bottom panel of Fig. 8 displays the autocorrelation function (ACF; Edelson \& Krolik 1988; Hufnagel \& Bregman 1992; Peterson et al. 2004) of the $R$-band data. The most remarkable feature is that it shows two prominent peaks at $\sim 7.6$ and $\sim 8.5 \mathrm{yr}$. The reason is that this method, which is more sensitive to maxima and minima brightness levels than to sinusoidal modulations of the light curves, feels the double-peaked nature of some events and the differences in the peak distances (see Fig. 7).

A similar plot for the $8 \mathrm{GHz}$ light curve, the most extended and best sampled among the radio datasets, is presented in Fig. 9 . The DFT has been obtained after binning the data over 1 week, thus making the sampling more homogeneous. The strongest signal corresponds to the $5.6 \mathrm{yr}$ period, which has not been suppressed by the lack of the predicted outburst in 2004, followed by signals at 3.7, 15.7, 8.0, 2.8, $1.9 \mathrm{yr}$, and by minor ones. We notice that some of the time scales are roughly multiples of the others, and that in the ACF plot the strongest peaks are found at about 11 and $15-17 \mathrm{yr}$, i.e. at twice the 5.6 and $8 \mathrm{yr}$ periods detected with the DFT. However, even for these peaks the value of the ACF does not exceed 0.5.

We also tested our results with the structure function (SF; Simonetti et al. 1985) method, and found that its results are very similar to those already obtained with the ACF.

An $\sim 8 \mathrm{yr}$ characteristic time scale of variability would put into correlation the outbursts occurred in the periods labelled $\alpha$, $\beta$, and $\gamma$ in Fig. 7. If this $\sim 8$ yr recurrence should repeat, an outburst will be visible during the 2006-2007 observing season.

We also performed a cross-correlation analysis by means of the discrete correlation function (DCF) on the historical $R$-band and radio light curves in order to check whether the addition of a further observing season of data can modify the 
results reported by Raiteri et al. (2005). Both the time lags of the radio variations with respect to the optical ones (from 1 to 2.5 months depending on the frequency separation) and the delays among variations at different radio frequencies (from 10 days to 1.5 months) found by Raiteri et al. (2005) are confirmed.

\section{Multiepoch spectral energy distribution}

The broad-band spectral energy distributions (SEDs) of AO $0235+164$ corresponding to the times when X-ray satellites observed the source are displayed in Fig. 10. Only epochs after 1990 were considered, thus neglecting the uncertain results obtained by Einstein in 1979-1981 and by EXOSAT in 1984. An analysis of the XMM-Newton and Chandra observations can be found in Raiteri et al. (2006), where a summary of previous $\mathrm{X}$-ray observations by other satellites is also given together with the pertaining references.

For the Chandra and XMM-Newton X-ray spectra, we display the results of both single power law (black bow-tie) and double power law (red line) models with Galactic absorption plus absorption from the intervening system at $z=0.524 \mathrm{ob}-$ tained by Raiteri et al. (2006). Those authors favoured the latter, curved solution. For all epochs but the February 2002 one, for which the curved solution was by far statistically superior, this was mainly motivated by the fact that in this energy region we expect the superposition of the synchrotron and inverseCompton components. We also display the UV and optical fluxes in the $M 2, W 1, U, B$, and $V$ bands derived by Raiteri et al. (2006) by analysing the observations of the Optical Monitor onboard XMM-Newton.

In the figure, optical (and near-IR) data simultaneous to the $\mathrm{X}$-ray observations are plotted with filled symbols; when simultaneous data were missing, we searched for the closest data available, and showed them with empty symbols. Since radio variations are slower than the optical ones, we plotted as filled symbols both strictly simultaneous radio data and data obtained as averages of those acquired just before and just after the satellite pointing.

Optical data were corrected for the ELISA contribution. Near-IR and optical magnitudes were de-reddened according to the prescriptions given by Junkkarinen et al. (2004), adopting the values tabulated by Raiteri et al. (2005), and then transformed into fluxes with the zeropoint values given by Bessell (1979).

Radio flux densities at $4.8,8.0$, and $14.5 \mathrm{GHz}$ are from UMRAO, those at 22 and $37 \mathrm{GHz}$ are from the Metsähovi Radio Observatory. RATAN simultaneous data at 0.9, 2.3, $3.9,4.8,7.7,11.1$, and $21.7 \mathrm{GHz}$ contributed to some SEDs (ASCA-Feb98, Chandra-Aug00, XMM-Feb02, XMM-Aug04). In the last three SEDs, corresponding to the XMM-Newton pointings inside the WEBT campaign, radio data at 4.9 and $10.5 \mathrm{GHz}$ from the Effelsberg telescope have been added.

The green solid line plotted in each panel represents a cubic spline interpolation through the August 2004 data; it was drawn only to help the comparison of SEDs.

As one can see from Fig. 10, the flat radio spectrum can always be connected with the steep optical one through what is called "the synchrotron bump". This is likely peaking in the farinfrared, as low-energy peaked BL Lac objects are expected to do. Most of the times the X-ray spectrum is hard, suggesting that we are seeing the first part of the inverse-Compton bump, which is expected to peak in the GeV energy range. However, in summer 1993 and February 2002 the X-ray spectrum is soft. As for the ROSAT spectrum, Madejski et al. (1996) suggested that in a bright state the peak of the synchrotron component may shift to higher energies, and thus the synchrotron radiation would dominate in the soft X-ray region. This interpretation was also followed by Padovani et al. (2004), when fitting the ROSAT spectrum with the homogeneous, one-zone model of Ghisellini et al. (2002). However, this scenario is questioned by the contemporaneous optical data shown in Fig. 10. Indeed, by looking at the various SEDs of this figure, there are no clues that the peak frequency may strongly change depending on the brightness state of the source. Actually, the situation may be more complex, since a UV-soft-X-ray bump, i.e. an extra SED component in addition to the synchrotron and inverse-Compton ones, seems to be present at least in some cases, as discussed by Junkkarinen et al. (2004) and Raiteri et al. (2005, 2006).

In the following, we briefly examine the individual SEDs, with particular attention to the optical data, since the presence of optical data simultaneous with the X-ray ones is fundamental to understand the emission mechanisms operating in the different energy bands.

\subsection{ROSAT: summer 1993}

The ROSAT pointing lasted from July 21 to August 15, 1993. The X-ray spectrum displayed as a blue, filled bow-tie in the figure refers to the analysis performed by Madejski et al. (1996) on the full dataset and reveals a bright and soft X-ray spectrum. However, since the ROSAT light curve showed significant variability, Comastri et al. (1997) reanalysed the data identifying two epochs of higher and lower count rates. The first epoch (July) yielded results similar to those of Madejski et al. (1996). $R$-band data taken on July 27 by the Hamburg Quasar Monitoring program ${ }^{10}$ are plotted in the figure as blue triangles. The connection of these data with the X-ray spectrum seems to require an extra bump.

The second ROSAT epoch (August) produced a different spectrum, which is plotted in the figure as a black, empty bow-tie. It is simultaneous with the acquisition of the $V$ and $R$ data displayed as black dots, which were taken at the Lowell Observatory on August 13. In this case the extra bump is not strictly needed, but also not ruled out.

\subsection{ASCA: February 1994 and 1998}

The first ASCA pointing (black, empty bow-tie) lasted from February 4 to 19, 1994 (Madejski et al. 1996). The closest optical data (black circles) were acquired at the JKT (La Palma) on January 13 (Takalo et al. 1998).

ASCA pointed at the source again on February 11-12, 1998, when it was in a bright radio and optical state (see Figs. 4 and 7). However, the X-ray flux was not particularly high, and the X-ray spectrum was harder (blue, filled bow-tie) than during the previous pointing (Junkkarinen et al. 2004). A simultaneous optical-UV spectrum was acquired with the HST (Junkkarinen et al. 2004), which is displayed as a blue line in Fig. 10, and simultaneous $B V R$ data were taken with the REOSC telescope of the Torino Observatory (blue squares), well matching the HST spectrum. The misalignment of the HST and X-ray spectra was discussed by Junkkarinen et al. (2004), who considered the possibility of an extra SED component as unlikely and suggested instead that it may arise from the difficulties in modelling the extinction of the $z=0.524$ absorber.

\footnotetext{
${ }^{10}$ http://www. friedensblitz.de/hqm/
} 

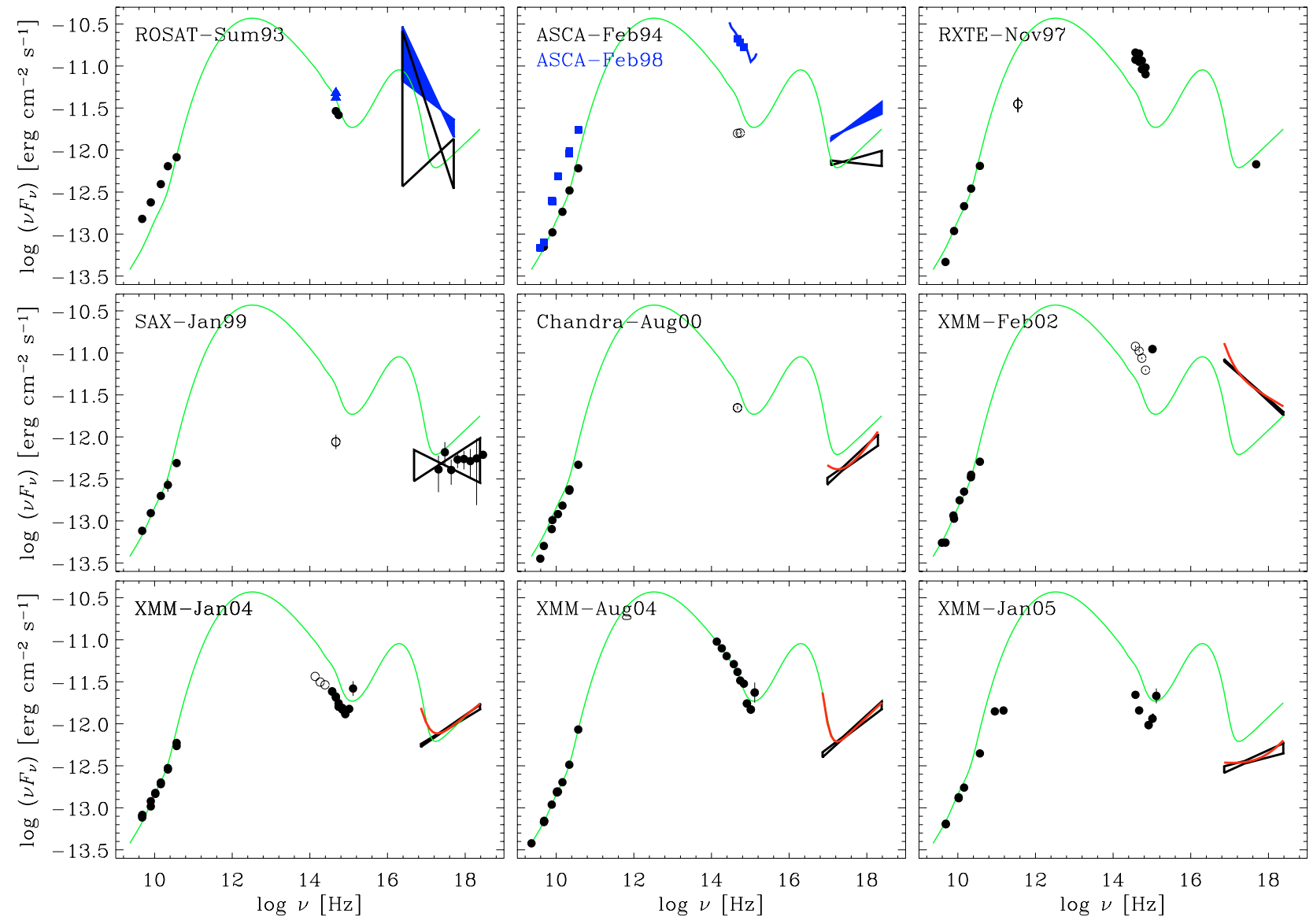

Fig. 10. Spectral energy distributions of AO $0235+164$ during X-ray satellite pointings. The green solid line represents a cubic spline interpolation through the August 2004 data.

It is interesting to point out here the correlation between the radio and X-ray spectral states: the harder and brighter X-ray spectrum of 1998 corresponds to a brighter and flatter radio spectrum, as expected when considering the inverse-Compton origin of the X-ray radiation.

\subsection{RXTE: November 1997}

The RXTE pointing was performed on November 3-6, 1997, during the rising phase of the optical outburst (see Fig. 4). The $\mathrm{X}$-ray flux was found low (Webb et al. 2000). Simultaneous optical data were taken by many observatories in the ambit of the first-light WEBT campaign (Raiteri et al. 2001). In Fig. 10 we plotted two $B V R I$ spectra obtained at the Teide Observatory on November 3 and 6 in order to have homogeneous data. Also shown is a point at $850 \mu \mathrm{m}$ obtained by averaging data taken at the JCMT before and after the RXTE observations (Webb et al. 2000).

\subsection{BeppoSAX: January 1999}

BeppoSAX observed AO $0235+164$ on January 28, 1999. The bow-tie in Fig. 10 shows the results of the analysis by Padovani et al. (2004), while the points in the X-ray domain were obtained directly by the ASI BeppoSAX Data Center ${ }^{11}$. The $R$-band datum was taken at the Torino Observatory 5 days before.

${ }^{11}$ http://www.asdc.asi.it/bepposax/

\subsection{Chandra: August 2000}

The Chandra observations were performed on August 20-21, 2000. They revealed a faint and hard X-ray spectrum (Turnshek et al. 2003; Raiteri et al. 2006). The closest optical datum was taken at the Torino Observatory 7 weeks later.

\subsection{XMM-Newton: February 2002}

The XMM-Newton observations of February 10, 2002 were analysed in detail by Raiteri et al. (2006) (see also Kadler 2005; Foschini et al. 2006). The source was found in the brightest X-ray state ever detected $\left(F_{1 \mathrm{keV}} \sim 2 \mu \mathrm{Jy}\right)$, with a soft spectrum, and the X-ray light curve revealed noticeable variability on a time scale of $\sim 40 \mathrm{~min}$. The Optical Monitor (OM) onboard XMM-Newton observed only in the UVW1 band, finding a bright UV state. Three $R$-band frames were acquired at the Mount Maidanak Observatory $\sim 1.5$ days before the XMM-Newton observations, revealing a moderately bright state $(R \sim 16.6)$. In the figure we plotted a complete $B V R I$ optical spectrum taken at Mount Maidanak 11 days earlier, when the source was exactly at the same optical level. However, an $I$-band datum taken at the Torino Observatory 9 days after the XMM-Newton pointing showed the source to be much fainter $(I \sim 16.9)$, so it is possible that at the time of the XMMNewton observation the source was in a dimming phase, and that simultaneous optical data would place just below those shown in the figure. In conclusion, it is very unlikely that the optical, $\mathrm{UV}$, and X-ray data can be fitted by a single convex synchrotron 
component. The UV-soft-X-ray bump would instead provide a comfortable way to describe this SED.

\subsection{XMM-Newton: January 2004}

The SED corresponding to the XMM-Newton pointing of January 18-19, 2004 was described in detail in Raiteri et al. (2005). The contemporaneous radio data come from Metsähovi, Effelsberg, and UMRAO, the optical (and near-IR) data were taken at the NOT, while the OM observed in the $V, B, U, U V W 1$, and $U V M 2$ filters. The transition from a steep optical spectrum to a flat UV one is dramatic, and the probable curvature of the X-ray spectrum supports the existence of the UV-soft-X-ray bump.

\subsection{XMM-Newton: August 2004}

The plotted radio points at 4.9 and $10.5 \mathrm{GHz}$ are averages of the intraday observations at Effelsberg (see Fig. 5); the $14.5 \mathrm{GHz}$ datum is from UMRAO; the $37 \mathrm{GHz}$ point represents the average of the Metsähovi data. Data at 2.3, 4.8, 7.7, 11.1, and 21.7 $\mathrm{GHz}$ taken simultaneously by RATAN at the beginning of September have been added to increase the sampling. They nicely agree with the other radio data. Near-IR information comes from Campo Imperatore. Many optical data are available in the $B V R I$ bands, with a fair sampling, showing a constant level without any significant variation. We display the averages of the data taken at Skinakas, to have a homogeneous BVRI set. Other $B V R I$ sequences acquired at Mount Maidanak just before the start of the XMM-Newton observations confirm the Skinakas average spectrum. The hints for the extra bump come from the UVM2 datum and the strong curvature of the X-ray spectrum when a double power law model is applied.

\subsection{XMM-Newton: January 2005}

As in the previous epoch, the points at 4.9 and $10.5 \mathrm{GHz}$ represent the averages of the Effelsberg data (see Fig. 6); the $14.5 \mathrm{GHz}$ datum is from UMRAO, the $37 \mathrm{GHz}$ one was obtained at Metsähovi, and the 2 and $3 \mathrm{~mm}$ data were acquired at Pico Veleta. In the optical band, the $I$ datum is from Mount Maidanak and the $R$ datum is from Boltwood. Here the curvature of the X-ray spectrum is smaller than in the previous epoch, but there are two UV points (UVW1 and UVM2 from the OM) that may support the existence of a UV bump.

In summary, the existence of the UV-soft-X-ray bump in the SED of AO $0235+164$ is supported by two arguments: the SED hardening in the UV domain, which gives rise to a concave optical-UV branch, and the corresponding "rise" of the soft-X-ray spectra towards the UV band. Even if the first argument should fall in case the UV extinction in the $z=0.524$ intervening system had been strongly overestimated by Junkkarinen et al. (2004), the extra bump cannot be avoided in the cases where the X-ray spectrum is soft.

\section{Discussion and conclusions}

Among blazars, AO $0235+164$ presents several peculiar features. It has shown dramatic flux changes at all wavelengths on a wide range of timescales, including IDV. Its main outbursts have repeated quasi-periodically, at least for some time, with clear correlation of the radio and optical flux variations with very short time delays. When considering this along with the presence of an intervening galactic system, also gravitational microlensing becomes a viable interpretation for the source variability. At milliarcsecond scales it shows an extremely compact core, with faint extensions occasionally appearing at various position angles. Several observations suggest that the source radiation is unusually highly Doppler-boosted.

In this paper we presented the results of the radio, near-IR, and optical monitoring during the second observing season of the WEBT campaign 2003-2005 targeting AO 0235+164. The results of the first observing season and a detailed analysis of the three XMM-Newton observations performed during the campaign have already been reported in Raiteri et al. $(2005,2006)$.

The big radio outburst, with a possible optical counterpart, which was foreseen to peak around February-March 2004 on the basis of the quasi-periodicity of $5.7 \pm 0.5 \mathrm{yr}$ discussed by Raiteri et al. (2001), did not occur. The radio flux remained rather low during the whole WEBT campaign, with some mild increased activity at the higher radio frequencies only in the last observing season 2004-2005. A mild increased activity in the last season also characterized the optical and near-IR light curves. The comparison of the optical and radio data taken during the WEBT campaign with the long-term light curves of this object shows how this last, long period of suppressed activity especially at radio wavelengths is unusual for this source. The analysis of the historical light curves reveals now a longer characteristic time scale of variability of $\sim 8 \mathrm{yr}$, more evident in the optical, but also present in the radio data along with the $\sim 5.7 \mathrm{yr}$ one.

The colour-index analysis reveals noticeable spectral changes in the optical band, which nonetheless do not show correlation with the source magnitude. The long-term trend appears essentially achromatic, with a possible progressive reddening in the last years.

All the three XMM-Newton observations performed during the WEBT campaign found the source in a faint state at all wavelengths. Neither the optical nor the X-ray light curves show any significant variability. In contrast, the simultaneous intraday radio observations at the $100 \mathrm{~m}$ Effelsberg telescope reveal some flux changes at $10.5 \mathrm{GHz}$. If they are ascribed to intrinsic processes, a Doppler factor up to $\sim 100$ can in principle be derived, confirming previous findings that this is an extremely beamed source. However, on the basis of these data we cannot rule out that interstellar scintillation was responsible for at least part of the variability. Actually, a reduced Doppler factor would agree with recent findings pointing to a reduced beaming during the current relatively quiescent period.

We compiled the SEDs corresponding to the X-ray observations since the ROSAT pointing in summer 1993. Radio and optical data simultaneous with the X-ray observations have been looked for in our database in order to shed light on the various emission components involved. The aim was mainly to find clues to the presence of the UV-soft-X-ray bump already discussed by Junkkarinen et al. (2004) and Raiteri et al. (2005, 2006). The existence of this extra component, besides the classical synchrotron and inverse-Compton ones, is mainly supported by the UV data, when available, and by the soft X-ray spectra detected by ROSAT in summer 1993 and by XMM-Newton in February 2002.

A discussion on the possible interpretations of this bump can be found in Raiteri et al. (2006). Among the various pictures, these authors focused mainly on the presence of a thermal accretion disc and on an additional synchrotron component. In the latter case, which was favoured by the authors, we can envisage two possibilities. Either there are actually two synchrotron 
components, coming from distinct and separate jet regions without any simple relationship, or we are in the presence of a continuous inhomogeneous jet whose radiation is for some reason suppressed in the optical-near-UV region. Here we have again two possibilities: i) the break in the spectrum is due to a discontinuity in the opacity (as in the case of Mkn 501, Raiteri \& Villata 2003), ii) the suppressed emission is caused by a misalignment of the corresponding jet region whose radiation is less beamed towards the line of sight ${ }^{12}$. The observed strong variability in both these SED components might favour a scenario where both the emission from the near-IR to the soft X-rays and the spectral gap change in time as the strongly bent jet precesses or rotates. This view may be supported by the large variations of the VLBI jet components position angles observed by many authors (see e.g. Frey et al. 2000, 2006; Jorstad et al. 2001; Piner et al. 2006).

We noticed how among the ten satellite pointings in 1993-2005, only two of them (ROSAT in summer 1993 and XMM-Newton in February 2002) found the source in a bright and soft X-ray state, possibly of synchrotron origin. The All Sky Monitor X-ray light curve of AO $0235+164^{13}$ from January 1996 to November 2005 shows that X-ray bright states are indeed very uncommon for this source: only in the first half of 2002 a significant increase of the count rate was observed, in correspondence to the first XMM-Newton pointing.

The two bright X-ray states are separated by about $8.5 \mathrm{yr}$, and a $\sim 9$ yr interval elapsed between the ROSAT and the 1984 EXOSAT observation, when a high (but very uncertain) X-ray flux was measured. A similar time separation is found when considering the last strongest optical outbursts of 1990 and 1998. Thus, both of them seem to follow an X-ray brightening by about $5 \mathrm{yr}$. It will be interesting to see whether an optical flux increase will occur in the next observing seasons, i.e. about $5 \mathrm{yr}$ after the 2002 X-ray bright state. It would support both the possible quasi-periodicity of $\sim 8 \mathrm{yr}$ of the optical (and radio) outbursts and a correlation between the optical and X-ray emission with a long time delay. A correlation of this type would be explained by a scenario where a curved inhomogeneous jet precesses or rotates, thus subsequently aligning its different emitting portions with the line of sight.

Several WEBT members are continuing to monitor the source in the radio, near-IR, and optical bands so that we will be able to check whether the source activity continues to increase and eventually leads to a forthcoming outburst.

Acknowledgements. We thank the staff of the Asiago Observatory for performing the optical observations of this paper in service mode and the staff of the Medicina Radio Station for assistance during the observations. Based on observations with the $100 \mathrm{~m}$ telescope of the MPIfR (Max-Planck-Institut für Radioastronomie) at Effelsberg. Based on observations made with the Nordic Optical Telescope, operated on the island of La Palma jointly by Denmark, Finland, Iceland, Norway, and Sweden, in the Spanish Observatorio del Roque de los Muchachos of the Instituto de Astrofísica de Canarias. Based on observations made with the Italian Telescopio Nazionale Galileo (TNG) operated on the island of La Palma by the Centro Galileo Galilei of the INAF (Istituto Nazionale di Astrofisica) at the Spanish Observatorio del Roque de los Muchachos of the Instituto de Astrofísica de Canarias. Based on observations made at Complejo Astronómico El Leoncito, operated under agreement among CONICET, UNLP, UNC, and UNSJ, Argentina. This research has made use of data from the University of Michigan Radio Astronomy Observatory, which is supported by the National Science Foundation and by funds from the University of Michigan. RATAN-600 observations were partly

\footnotetext{
12 Although at other wavelengths (and hence spatial resolution), this scenario has indeed been suggested by Bach et al. (2006) to interpret the region of suppressed flux density in the intensity profiles of the VLBA images of BL Lacertae.

13 http://heasarc.nasa.gov/docs/xte/ASM/sources.html
}

supported by the Russian State Program "Astronomy" (project 1.2.5.1), the Russian Ministry of Education and Science, and the Russian Foundation for Basic Research (projects 01-02-16812, 02-02-16305, 05-02-17377). This work was partly supported by the European Community's Human Potential Programme under contract HPRN-CT-2002-00321 (ENIGMA) and by the Italian Space Agency under contract ASI-INAF I/023/05/0. V.M.L. acknowledges support from Russian Federal Foundation for Basic Research (RFBR), project 05-02-17562. MK has been supported in part by a Fellowship of the International Max Planck Research School for Radio and Infrared Astronomy at the Universities of Bonn and Cologne and in part by an appointment to the NASA Postdoctoral Program at the Goddard Space Flight Center, administered by Oak Ridge Associated Universities through a contract with NASA. This project was done while Y.Y.K. was a Jansky fellow of the National Radio Astronomy Observatory and a research fellow of the Alexander von Humboldt Foundation.

\section{References}

Bach, U., Villata, M., Raiteri, C. M., et al. 2006, A\&A, 456, 105 Bessell, M. S. 1979, PASP, 91, 589

Blandford, R. D., \& Königl, A. 1979, ApJ, 232, 34

Blandford, R. D., \& Rees, M. J. 1978, in Pittsburgh Conference on BL Lac Objects, Pittsburgh, Pa., April 24-26, Proceedings, 328

Böttcher, M., Harvey, J., Joshi, M., et al. 2005, ApJ, 631, 169

Comastri, A., Fossati, G., Ghisellini, G., \& Molendi, S. 1997, ApJ, 480, 534

Edelson, R. A., \& Krolik, J. H. 1988, ApJ, 333, 646

Fiorucci, M., Tosti, G., \& Rizzi, N. 1998, PASP, 110, 105

Fiorucci, M., Ciprini, S., \& Tosti, G. 2004, A\&A, 419, 25

Foschini, L., Ghisellini, G., Raiteri, C. M., et al. 2006, A\&A, 453, 829

Frey, S., Gurvits, L. I., Altschuler, D. R., et al. 2000, PASJ, 52, 975

Frey, S., Gurvits, L. I., Gabuzda, D. C., et al. 2006, PASJ, 58, 217

Fujisawa, K., Kobayashi, H., Wajima, K., et al. 1999, PASJ, 51, 537

Ghisellini, G., Celotti, A., \& Costamante, L. 2002, A\&A, 386, 833

Gu, M., Lee, C.-U., Pak, S., Yim, H. S., \& Fletcher, A. B. 2006, A\&A, 450, 39

Hufnagel, B. R., \& Bregman, J. N. 1992, ApJ, 386, 473

Jorstad, S. G., Marscher, A. P., Mattox, J. R., et al. 2001, ApJS, 134, 181

Junkkarinen, V. T., Cohen, R. D., Beaver, E. A., et al. 2004, ApJ, 614, 658

Kadler, M. 2005, Ph.D. Thesis, MPIfR, Bonn

Kovalev, Y. Y., Kellermann, K. I., Lister, M. L., et al. 2005, AJ, 130, 2473

Kraus, A., Quirrenbach, A., Lobanov, A. P., et al. 1999, A\&A, 344, 807

Kraus, A., Krichbaum, T. P., Wegner, R., et al. 2003, A\&A, 401, 161

Madejski, G., Takahashi, T., Tashiro, M., et al. 1996, ApJ, 459, 156

Ostorero, L., Villata, M., \& Raiteri, C. M. 2004, A\&A, 419, 913

Ostriker, J. P., \& Vietri, M. 1985, Nature, 318, 446

Padovani, P., Costamante, L., Giommi, P., et al. 2004, MNRAS, 347, 1282

Peterson, B. M., Ferrarese, L., Gilbert, K. M., et al. 2004, ApJ, 613, 682

Piner, B. G., Bhattarai, D., Edwards, P. G., \& Jones, D. L. 2006, ApJ, 640, 196

Press, W. H., Teukolsky, S. A., Vetterling, W. T., \& Flannery, B. P. 1992, Numerical recipes in FORTRAN. The art of scientific computing (Cambridge: University Press), 2nd edn.

Raiteri, C. M., \& Villata, M. 2003, in Proc. of the First ENIGMA Meeting, held at Mayschoss, Germany, May 11-14, ed. M. Hauser, U. Bach, \& S. Britzen, 326

Raiteri, C. M., Villata, M., Aller, H. D., et al. 2001, A\&A, 377, 396

Raiteri, C. M., Villata, M., Ibrahimov, M. A., et al. 2005, A\&A, 438, 39

Raiteri, C. M., Villata, M., Kadler, M., et al. 2006, A\&A, 452, 845

Rickett, B. J. 1990, ARA\&A, 28, 561

Romero, G. E., Fan, J.-H., \& Nuza, S. E. 2003, Chin. J. Astron. Astrophys., 3, 513

Sillanpää, A., Haarala, S., Valtonen, M. J., Sundelius, B., \& Byrd, G. G. 1988, ApJ, 325, 628

Simonetti, J. H., Cordes, J. M., \& Heeschen, D. S. 1985, ApJ, 296, 46

Smith, P. S., Balonek, T. J., Heckert, P. A., Elston, R., \& Schmidt, G. D. 1985, AJ, 90, 1184

Stickel, M., Fried, J. W., \& Kuehr, H. 1988, A\&A, 198, L13

Takalo, L. O., Sillanpää, A., Valtaoja, E., et al. 1998, A\&AS, 129, 577

Turnshek, D. A., Rao, S. M., Ptak, A. F., Griffiths, R. E., \& Monier, E. M. 2003, ApJ, 590, 730

Villata, M., \& Raiteri, C. M. 1999, A\&A, 347, 30

Villata, M., Raiteri, C. M., Sillanpaa, A., \& Takalo, L. O. 1998, MNRAS, 293, L13

Villata, M., Raiteri, C. M., Kurtanidze, O. M., et al. 2002, A\&A, 390, 407

Villata, M., Raiteri, C. M., Aller, H. D., et al. 2004a, A\&A, 424, 497

Villata, M., Raiteri, C. M., Kurtanidze, O. M., et al. 2004b, A\&A, 421, 103

Villata, M., Raiteri, C. M., Balonek, T. J., et al. 2006, A\&A, 453, 817

Wagner, S. J., Witzel, A., Heidt, J., et al. 1996, AJ, 111, 2187 
Webb, J. R., Howard, E., Benítez, E., et al. 2000, AJ, 120, 41

Zensus, J. A., Ros, E., Kellermann, K. I., et al. 2002, AJ, 124, 662

1 INAF, Osservatorio Astronomico di Torino, 10025 Pino Torinese, Italy

e-mail: raiteri@to.astro.it

2 Max-Planck-Institut für Radioastronomie, 53121 Bonn, Germany

${ }^{3}$ NASA/Goddard Space Flight Center, Code 662, Greenbelt, Maryland 20771, USA

${ }^{4}$ Ulugh Beg Astron. Inst., Academy of Sciences of Uzbekistan, Tashkent 700052, Uzbekistan

5 Abastumani Observatory, 383762 Abastumani, Georgia

6 Astron. Inst., St.-Petersburg State Univ., 198504 St.-Petersburg, Russia

7 Metsähovi Radio Observatory, Helsinki Univ. of Technology, 02540 Kylmälä, Finland

81655 Stittsville Main St., Stittsville, Ont., K2S 1N6, Canada

9 Korea Astronomy and Space Science Institute, Republic of Korea

10 Dept. of Astronomy, Univ. of Michigan, Ann Arbor, MI 48109, USA

11 Inst. Argentino de Radioastronomía, 1894 Villa Elisa, Argentina

12 Facultad de Ciencias Astronómicas y Geofísicas, UNLP, La Plata, Argentina

13 Pulkovo Observatory, St. Petersburg, Russia

14 Dept. of Physics \& Astronomy, Western Kentucky Univ., Bowling Green, KY 42104, USA

15 Tuorla Observatory, 21500 Piikkiö, Finland

16 INAF, Osservatorio Astrofisico di Catania, 95123 Catania, Italy
17 Armenzano Astronomical Observatory, Assisi, Italy

18 Steward Observatory, 933 N.Cherry Ave. Tucson, AZ 85721, USA

19 INAF, Osservatorio Astronomico di Collurania Teramo, 64100 Teramo, Italy

20 Dipartimento di Fisica e Osservatorio Astronomico, Università di Perugia, Italy

21 Astrophysikalisches Institut Potsdam, 14482 Potsdam, Germany

22 Argelander Institut für Astronomie, Universität Bonn, 53121 Bonn, Germany

23 National Radio Astronomy Observatory, Green Bank, WV 24944, USA

24 Astro Space Center of Lebedev Physical Inst., 117997 Moscow, Russia

25 INAF, Istituto di Radioastronomia, Sezione di Noto, 96017 Noto, Italy

26 Dept. of Natural Sciences, Fayetteville State Univ., Fayetteville, NC 28301, USA

27 INAF, Osservatorio Astronomico di Roma, 00040 Monte Porzio Catone, Italy

28 Michael Adrian Observatory, 65468 Trebur, Germany

29 IESL, FORTH, 71110 Heraklion, Crete, Greece

30 Physics Dept., Univ. of Crete, 71003 Heraklion, Crete, Greece

31 Nordic Optical Telescope, 38700 Santa Cruz de La Palma, Spain

32 Akhaltsikhe branch of the Tbilisi State University, Georgia

33 IRAM, Avd. Div. Pastora 7NC, 18012 Granada, Spain

34 Inst. of Astronomy and Astrophysics, National Observatory of Athens, 11810 Athens, Greece 